\title{
A Technique of Distal Clavicle Fracture Fixation Using The Tightrope Procedure
}

\author{
CJ Soh, MS Orth (Mal), N Sivapathasundaram, MS Oth (Mal), Rensgsen Parthiban, MS Orth (Mal), \\ A Ramanand, MBBS (MMMC)
}

Department of Orthopaedic Surgery, Hospital Melaka, Melaka, Malaysia

\begin{abstract}
We present here a technique of fracture stabilization using the Tightrope procedure in a patient with a widely displaced Neer type IIB distal clavicle fracture. The Tightrope system, typically used for stabilization of acromioclavicular joint dislocation, has not been widely described for distal clavicle fractures. The patient achieved satisfactory results after surgery; we feel that this technique is appealing as it is simple, reproducible and avoids the complications associated with extensive metalwork. This technique may also appeal to the arthroscopic surgeon.
\end{abstract}

Key Words:

Neer Type Ii Distal Clavicle Fracture, Tightrope Procedure, Arthroscopy

\section{INTRODUCTION}

Distal clavicle fractures are common injuries and account for 10 to $15 \%$ of all clavicle fractures ${ }^{1,2}$. Neer has classified these fractures into three types, with type IIB being notoriously difficult to treat due to wide displacement of the proximal fragment ${ }^{2}$. Surgery is frequently needed, as there is a high rate of nonunion with conservative treatment. Most surgical techniques depend on extensive metalwork such as plates or wires, while some tend to violate normal structures such as the acromioclavicular joint. The Tightrope system, typically used for acromioclavicular joint dislocation, may also be a viable option for distal clavicle fracture stabilization.

\section{CASE REPORT}

A 24-year old female was involved in a motor vehicle accident and sustained a widely displaced left distal clavicle fracture with a small distal fragment attached to the acromion; this was classified as a Neer Type IIB distal clavicle fracture (Figure 1a). Surgery was performed on the second day after the injury.

General anaesthesia was administered and the patient was placed in a beach-chair position. A $6 \mathrm{~cm}$ incision extending from the coracoid process vertically to the distal clavicle was made; then the delto-trapezial interval was split longitudinally and delto-trapezial fascia separated from the clavicle to expose the fracture site. Inferiorly, the coracoid process was identified and the fracture site was cleared of interposed soft tissue. The small distal fragment of the fracture was noted to be still attached to acromion and was retained, but the trapezoid and conoid components of the coracoclavicular ligament were ruptured.

Reduction was carried out using downward pressure on the proximal fragment and a reduction clamp was applied to maintain temporary reduction. Using a Tightrope guide placed at the superior surface of the distal end of the proximal fragment and inferiorly over the under surface of the base of the coracoid process, a $2.4 \mathrm{~mm}$ guide pin was placed across both cortices of the clavicle and coracoid process. Care was taken to ensure that the guide pin was well centred over the base of the coracoid process to avoid breaching through the sides during drilling. A $4 \mathrm{~mm}$ cannulated drill was advanced across the pin and drilling was performed from the clavicle to the base of the coracoid process. Two tunnels of $4 \mathrm{~mm}$ in diameter were thus created in each of the proximal fragments of the distal clavicle fracture and coracoid process.

An implant passer was inserted from superiorly over the clavicle through both the tunnels. The Tightrope (Arthrex, Naples) implant was inserted into the clavicular and coracoid tunnel using an implant pusher; first the oval metallic button was advanced in a vertical position, and this was followed by the fibrewires. Once the pusher was fully advanced, it was withdrawn together with the passer. This action prompts the oval metallic button to flip into horizontal position, thus engaging the coracoid base. The fibrewires were alternately pulled to place the round metallic button flat on the superior surface of the distal clavicle tunnel. After satisfactory tension was achieved, knots were made on top of the round button to complete the fixation (Figure 2). The reduction clamp was then removed followed by repair of trapezoid and conoid components of the coracoclavicular ligament and closure of the wound in layers. 


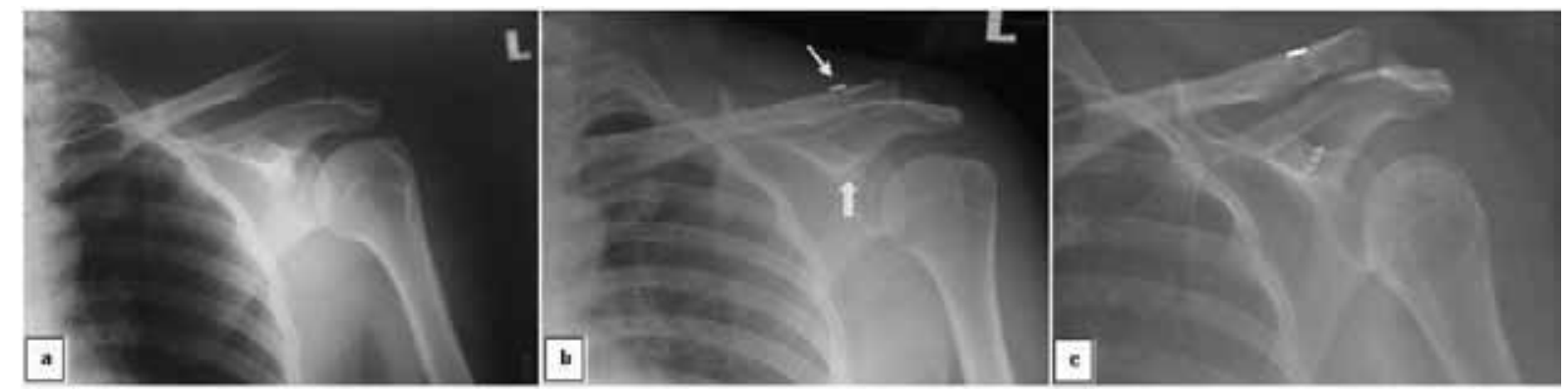

Fig. 1: a) Pre operative radiograph b) Immediate post operative radiograph with the Tightrope implant (thin arrow. round button, thick arrow. oval button) c) 12 weeks post operative radiograph.


Fig. 2: a) Intraoperative picture showing the fractured distal clavicle b) Fracture reduced with the Tightrope implant (round button on the clavicle).



Fig. 3: Post operative clinical pictures demonstrating good shoulder range of motion. 
The immediate post-operative radiograph revealed a reduced fracture and well positioned Tightrope implant (Figure 1b). The patient was instructed to use an arm sling for 4 weeks and perform gentle range of motion up to shoulder level for that period of time. Full range of motion of the shoulder and strengthening exercises were prescribed after 4 weeks. At 12 week follow-up, the patient was pain-free and had regained full range of movement of the left shoulder (Figure 3). Radiograph at 12 weeks revealed a well united fracture.

\section{DISCUSSION}

Neer type IIB distal clavicle fractures are characterized by disruption of the coracoclavicular ligament with wide proximal fragment displacement. There is a high risk of nonunion, ranging from 22 to $30 \%$ for nonoperative treatment ${ }^{2}$. Surgical treatment is usually indicated, as it is difficult to establish and maintain reduction with more conservative measures ${ }^{3}$. Various techniques have been described for treatment of these fractures, but the ideal method of fixation remains to ascertained ${ }^{3,4}$. This is probably due to the associated complications of many of these surgical techniques.

Originally designed for syndesmotic injury of the ankle, the Tightrope system has been used for anterior cruciate ligament reconstruction and stabilization of acromioclavicular joint dislocation. To the best of our knowledge, there is only one published study regarding the use of the Tightrope system for distal clavicle fracture ${ }^{5}$. The system has been used as an adjunct for closing the coracoclavicular interval in addition to fixation with plates. The Tightrope system consists of four-strand \#5 fibrewire interlaced between two titanium endobuttons. The so-called 'snow-shoe' hold of these buttons on cortical bone enables the implant to withstand cyclic load without cutting out of bone. This type of fixation provides good apposition of the fracture fragments for union by maintaining the reduced coracoclavicular interval. This also allows the injured coracoclavicular ligaments to heal better with anatomical reduction. As the acromioclavicular joint is not violated, complications such as arthrosis are avoided. There is no risk of shoulder impingement as no hardware is placed into the subacromial space as may occur with use of a hook plate. The implant is relatively low profile, and thus obviates the need for a second surgery for its removal. Another advantage of this technique is that it may be performed arthroscopically.

The main drawback of this technique is that the coracoid process must be exposed thereby placing the nearby musculocutaneous nerve at risk. Cost may be another consideration when compared to other cheaper alternatives.

There is a need for fixation method that offers adequate stability for early shoulder mobilization, yet avoids complications associated with conventional fixation methods. The Tightrope system is a promising option for displaced distal clavicle fractures as a stand-alone fixation. 


\section{REFERENCES}

1. Neer CS Jr. Fracture of the distal clavicle with detachment of coracoclavicular ligaments in adults. J Trauma. 1963; 3: 99-110.

2. Neer CS 2nd. Fractures of the distal third of the clavicle. Clin Orthop.1968; 58: 43-50.

3. Kao F-C, Chao E-K, Chen C-H, Chen CY, Yen CY. Treatment of distal clavicle fracture using Kirschner wires and tensionband wires. J Trauma. 2001; 51: 522-5.

4. Tambe AD, Motkur P, Qamar A, Drew S, Turner SM. Fractures of the distal third of the clavicle treated by hook plating. Int Orthop. 2006; 30: 7-10.

5. Yeow WL, Aman S, Roger P. Acromioclavicular joint reduction, repair and reconstruction using metallic buttons - early results and complications. Techniques in Shoulder \& Elbow Surgery. 2007; 8(4): 213-21. 